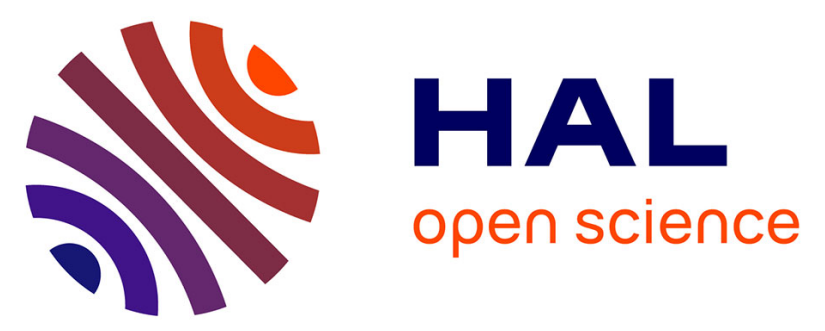

\title{
VARIATIONS STRUCTURELLES DES SYSTÈMES VOCALIQUES EN FRANÇAIS ET ESPAGNOL SOUS L'EFFET DU STYLE DE PAROLE
}

D. Poch-Olive, B. Harmegnies

\section{- To cite this version:}

D. Poch-Olive, B. Harmegnies. VARIATIONS STRUCTURELLES DES SYSTÈMES VOCALIQUES EN FRANÇAIS ET ESPAGNOL SOUS L'EFFET DU STYLE DE PAROLE. Journal de Physique IV Proceedings, 1992, 02 (C1), pp.C1-283-C1-286. 10.1051/jp4:1992160 • jpa-00251231

HAL Id: jpa-00251231

https://hal.science/jpa-00251231

Submitted on 1 Jan 1992

HAL is a multi-disciplinary open access archive for the deposit and dissemination of scientific research documents, whether they are published or not. The documents may come from teaching and research institutions in France or abroad, or from public or private research centers.
L'archive ouverte pluridisciplinaire HAL, est destinée au dépôt et à la diffusion de documents scientifiques de niveau recherche, publiés ou non, émanant des établissements d'enseignement et de recherche français ou étrangers, des laboratoires publics ou privés. 


\title{
VARIATIONS STRUCTURELLES DES SYSTĖMES VOCALIQUES EN FRANÇAIS ET ESPAGNOL SOUS L'EFFET DU STYLE DE PAROLE
}

\author{
D. POCH-OLIVE et B. HARMEGNIES* \\ Laboratori de fonètica, Facultat de Lletres, Universitat Autonoma de Barcelona, SP-08193 Bellaterra \\ (Barcelona), Spain \\ *Service de la Communication Parlée, Université de Mons, 20 place du Parc, B-7000 Mons, Belgium
}

\section{ABSTRACTS}

The paper analyzes utterances of the French and Spanish vowels both in spontaneous- and laboratory speech. These changes in speaking styles modify the vowels formants. In spanish, both centralization and increased probability of inter vowel confusion are observed. In French, decreased differenciation is observed as well, but without clear centralization tendencies.

La communication analyse des réalisations de voyelles françaises et espagnoles en parole spontanée et en parole de laboratoire. Ces changements de style modifient les formants vocaliques. En espagnol, on constate à la fois centralisation et tendance à la confusion entre voyelles. En français, une baisse de la différenciation est également observable, mais en l'absence de tendances centralisantes nettes.

\section{INTRODUCTION}

Divers travaux, tant théoriques qu'empiriques ont souligné que le changement de style de parole produit d'importantes variations des réalisations sonores. Les aspects qualitatifs et quantitatifs des variations rapportées semblent cependant variables d'une publication à l'autre, sinon contradictoires. Peu d'études ont en outre été menées sur les langues du groupe roman. Les recherches présentées ici se veulent une contribution à la clarification de cette question. Elles abordent le cas de deux langues romanes, l'une à système vocalique simple: l'espagnol castilian (nord-ouest de l'Espagne), et l'autre à système vocalique complexe: le français (variêté du sudouest de la Belgique).

\section{PROCEDURE EXPERIMENTALE.}

Dans le but de maximiser la variabilité liée au style de parole, nous avons recouru à deux formes très contrastées de situations de communication: la parole spontanée (conversation ordinaire), d'une part, et la parole de laboratoire (lecture de listes de mots), d'autre part.

Les échantillons de parole spontanée furent recueillis à partir d'entretiens informels entre l'un d'entre nous (DP, pour l'espagnol, et BH pour le français) et l'un des deux sujets étudiés ici. Ces derniers étaient chacun des locuteurs natifs monolingues (l'un francophone, l'autre hispanophone), de sexe masculin, âgés d'une trentaine d'années. Chaque conversation dura, en moyenne, 45 minutes, et prit la forme d'un entretien semi-directif où chaque sujet fut prié 
d'évoquer divers thèmes proches de sa vie privée.

Chacun des enregistrements fut ensuite manuellement retranscrit. Pour chacune des deux langues, la procédure décrite ci-après fut ensuite utilisée. Un ensemble d'une vingtaine de réalisations de chacune des voyelles fut sélectionné aléatoirement. Une liste des mots contenant ces voyelles fut ainsi dressée. Quelques jours après le premier enregistrement, le locuteur fut prié de réaliser des lectures de listes de mots. Cette session se déroula en chambre sourde; le locuteur était assis devant un moniteur vidéo qui affichait à un rythme régulier, un par un, les mots préalablement sélectionnés. Ceux-ci étaient en fait prélevés en ordre aléatoire dans la liste issue de l'enregistrement de parole spontanée.

Les enregistrements en espagnol furent réalisés à l'Universitat Autõnoma de Barcelona et au Service de la Communication Parlée, de l'Université de Mons. Les mesures acoustiques portèrent sur les formants des voyelles prises en considération. Leurs valeurs fréquentielles furent estimées au centre et aux frontières des voyelles, au moyen d'analyses spectrographiques et spectrales, réalisées sur une console KAY DSP 5500, avec une résolution constante d'au moins $10 \mathrm{~Hz}$.

Les deux bases de données résultant de ces analyses (une pour chaque langue) sont organisées en sorte de lier chaque réalisation d'une voyelle dans un style de parole à la réalisation de la voyelle issue du même mot dans l'autre style de parole. Notre unité d'analyse est donc la paire de sons, plutôt que le son, ce qui nous permettra de réaliser des analyses statistiques sur base d'observations pairées inter style. Dans cet article, nous limiterons, par manque d'espace, notre investigation aux fréquences formantiques des premier et second formants, évaluées au centre de ceux-ci.

\section{RESULTATS}

De nombreuses publications font état de réduction des timbres vocaliques à celui de la voyelle neutre. Nous tenterons ici de déterminer si cette tendance est observable, dans les deux langues étudiées, sous l'effet du changement de style. Nous utiliserons, à cet effet, l'indice $\delta$ (Harmegnies et Poch, 1992). Celui-ci repose sur l'estimation des distances euclidiennes entre les réalisations étudiēes et schwa:

$$
\delta=\left[\left(F_{1}-500\right)^{2}+\left(F_{2}-1500\right)^{2}\right]^{\frac{1}{2}}-\left[\left(f_{1}-500\right)^{2}+\left(f_{2}-1500\right)^{2}\right]^{\frac{1}{2}}
$$

avec $F_{1}$ et $F_{2}$ les premier et second formants de la voyelle en parole de laboratoire et $f_{1}$ et $f_{2}$ les premier et second formants de la voyelle en parole spontanée. L'indice $\delta$ présente des valeurs nulles si la position de la réalisation dans l'espace F1/F2 est invariante, et positives si la réalisation en parole spontanée est plus proche de schwa que la réalisation en parole de laboratoire.

Les tableaux 1 et 2 présentent des résumés statistiques des distributions de l'indice $\delta$ pour chaque voyelle, dans chacune des deux langues.

\begin{tabular}{crrr}
\hline voyelle & m & \multicolumn{1}{c}{$\sigma$} & N \\
\hline /i/ & 155.6110 & 92.9369 & 20 \\
lel & 230.4406 & 125.0558 & 20 \\
lal & 15.4158 & 110.9498 & 20 \\
lol & 101.3282 & 162.9051 & 20 \\
/u/ & 48.2482 & 115.9172 & 20 \\
\hline
\end{tabular}

Tableau 1: moyennes $(m)$, écarts types $(\sigma)$ et effectifs $(N)$ des indices $\delta$, calculés sur base des enregistremens hispanophones.

Ainsi que le montre le tableau 1, les enregistrements hispanophones sont dominés par une 
tendance sensible à la centralisation des voyelles. Les valeurs de $\delta$ sont en effet toutes positives et assez élevées. On notera cependant un renforcement de la tendance pour les voyelles d'avant, par opposition aux voyelles d'arrière; ainsi qu'un minimum de centralisation pour /a/.

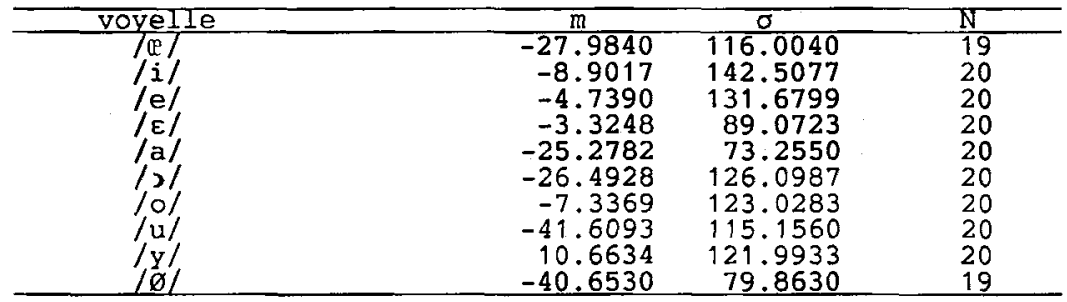

Tableau 2: moyennes $(m)$, écarts types $(\sigma)$ et effectifs $(N)$ des indices $\delta$, calculés sur base des enregistremens francophones.

Dans le cas du français (cfr tableau 2), la situation est cependant extrêmement différente. D'une part, la plupart des valeurs sont négatives: on ne constate donc pas, dans le chef des voyelles françaises, la même tendance à la centralisation que présentaient les voyelles espagnoles. Il est cependant clair que les réalisations se modifient sous l'effet du style, les valeurs de $\delta$ étant non nulles. D'autre part, les valeurs de l'indice semblent globalement plus faibles en français qu'en espagnol.

Les observations précédentes suggèrent que les agrégats formés par les réalisations vocaliques se meuvent dans le plan F1/F2 au passage de la parole de laboratoire à la parole spontanée. Dans le cas de l'espagnol, ces mouvements apparaissent converger vers une zone correspondant à schwa; cette tendance n'est pas observable en français. Ces constatations ne renseignent cependant pas directement sur l'éventuelle dégradation de la différenciation inter agrégats que pourrait produire le changement de style.

Dans le cas de l'espagnol, la convergence observée des agrégats suggère l'accroissement du risque de chevauchement entre les aires de répartition. Il s'avère également que les écarts types des valeurs formantiques sont systématiquement plus grands en parole spontanée qu'en parole de laboratoire (données non présentées ici). Nous avons cherché à tester cette hypothèse au moyen d'analyses discriminantes. L'ensemble des mesures de parole de laboratoire a ainsi été analysê, les formants étant considérés comme variables discriminantes, et les catégories vocaliques comme variables de groupement. Une fois obtenues les fonctions discriminantes, nous avons simulé une tâche de reconnaissance, consistant à associer chaque observation à une catégorie vocalique. Cette tentative produisit $99 \%$ de reconnaissances correctes, tous les sons étant correctement classés dans leur catégorie réelle, à l'exception d'un /o/ erronément identifié comme $/ \mathrm{u} /$.

La même procédure fut ensuite appliquée à la parole spontanée espagnole. Le score global de reconnaissance correcte chuta, dans ce cas, à $84 \%$. Le tableau 3 présente la matrice de confusion résultant de ce traitement.

\begin{tabular}{|c|c|c|c|c|c|}
\hline & 717 & $7 e 7$ & $1 \mathrm{al}$ & 101 & $7 \mathbf{u} 1$ \\
\hline $\begin{array}{l}\text { lil } \\
\text { lel } \\
\text { /al } \\
\text { lo/ } \\
\text { /ul }\end{array}$ & $\begin{array}{l}\frac{95}{5} \\
- \\
- \\
-\end{array}$ & $\begin{array}{r}5 \\
85 \\
5 \\
10 \\
-\end{array}$ & $\begin{array}{r}5 \\
65 \\
5\end{array}$ & $\begin{array}{r}\overline{5} \\
30 \\
80 \\
5\end{array}$ & $\begin{array}{r}- \\
- \\
\overline{5} \\
95\end{array}$ \\
\hline
\end{tabular}

Tableau 3: matrice de confusion issue de l'analyse discriminante des voyelles espagnoles en parole spontanée. Les valeurs, en pourcentages, expriment la quantité de sons relevant de chaque catégorie réelle (1ignes) associés à chaque catégorie prédite (colonnes).

Comme le montre ce tableau, certaines voyelles semblent très affectées par le 
changement de style: tel est le cas de /a/. Il importe en outre de noter que cette dégradation n'est pas directement liée au phênomène de centralisation précédemment observé: ainsi, par exemple, la voyelle /a/, qui présente ici la plus importante dégradation était la voyelle la moins centralisée $(\delta=15.4)$. On remarquera aussi que $/ i$ / et $/ u /$, caractérisés par des indices $\delta$ dans un rapport du simple au triple, connaissent cependant une perte de discriminabilité égale (et d'ailleurs réduite: $5 \%$ ).

La même procédure a été appliquée aux voyelles du français. Les taux de reconnaissance correcte s'élèvent à $58.6 \%$ (parole spontanée) et $\mathbf{8 0 . 3 \%}$ (parole de laboratoire). En parole de laboratoire, les taux de reconnaissance voyelle par voyelle varient de $70 \%$ a $100 \%$, avec une tendance centrale marquée à $80 \%$; le tableau 4 donne, par ailleurs, la matrice de confusion relative à la parole spontanée.

\begin{tabular}{|c|c|c|c|c|c|c|c|c|c|c|}
\hline & 7]e] & 7il & $\mathrm{Tel}$ & $|\varepsilon|$ & $7 \mathrm{a}$ & 731 & 701 & $7 \mathrm{ul}$ & $\mid \mathrm{z} /$ & 781 \\
\hline $\operatorname{lel}$ & 42.1 & & 15.8 & 21.1 & - & 5.3 & - & - & $\overline{5}$ & 15.8 \\
\hline $\begin{array}{l}\text { lil } \\
\text { lel }\end{array}$ & $2 \overrightarrow{5}$ & $\frac{95}{10}$ & $5 \overline{0}$ & $1 \overline{5}$ & $\bar{z}$ & $\overline{-}$ & $\overline{-}$ & $\overline{-}$ & $\begin{array}{l}5 \\
-\end{array}$ & $\overline{-}$ \\
\hline$|\varepsilon|$ & 40 & 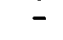 & 15 & 10 & 25 & 10 & - & - & - & - \\
\hline /al & $\overline{5}$ & $\overline{-}$ & $\stackrel{5}{-}$ & $\overline{-}$ & $\frac{95}{5}$ & $\overrightarrow{75}$ & $1 \overline{0}$ & $\overline{5}$ & $\overline{-}$ & $\overline{-}$ \\
\hline $10 \%$ & & & - & - & - & $\overline{25}$ & 50 & 25 & - & - \\
\hline $\mathrm{u}$ & & $2 \overline{0}$ & $\overline{-}$ & $=$ & - & $=$ & 20 & $\frac{80}{-}$ & $5 \overline{0}$ & $\overline{5}$ \\
\hline $\mid \begin{array}{l}y \\
|0|\end{array}$ & & & 10.5 & - & - & 5.3 & 5 & - & $\frac{\pi}{36}$ & 36.8 \\
\hline
\end{tabular}

Tableau 4: matrice de confusion issue de l'analyse discriminante des voyelles françaises en parole spontanée. Les valeurs, en pourcentages, expriment la quantité de sons relevant de chaque catégorie réelle (lignes) associés à chaque catégorie prédite (colonnes).

On y constate que les voyelles $/ \propto /, / \mathrm{e} /, / \mathrm{e} /, / \mathrm{o} /, / \mathrm{y} /$ et $/ \varnothing /$ présentent de taux de reconnaissance correcte inférieurs ou égaux à 50\%; les voyelles aux extrémités de l'espace $\mathrm{F} 1 / \mathrm{F} 2(/ \mathrm{i} /, / \mathrm{a} /, / \mathrm{u} /)$ sont cependant bien reconnues (95\%, 95\%, 80\%). Il apparaît donc que les transformations des agrégats vocaliques sous l'effet du style conduisent en français également à des variations du degré d'organisation du système vocalique. Notons au passage qu'une analyse plus fine gagnerait sans doute à intégrer -au moins dans cette langue- les valeurs du troisième formant. On peut raisonnablement penser que ce surcrôit d'information améliorerait les scores de reconnaissance dans les deux styles. Il semble cependant peu probable que la prise en compte de ces données modifie sensiblement le rapport de structuration des systèmes vocaliques dans les deux types de styles.

\section{CONCLUSION}

Les données présentées ici suggèrent qu'en parole de laboratoire, les systèmes vocaliques F1/F2 présentent des degrés d'entropie moins importants qu'en parole spontanée. Cette analyse des phénomènes mis en évidence pourrait s'expliquer par le fait que dans un style (spontané), l'information situationnelle (proxémique, sémantique, contextuelle, etc.) est abondante, alors que dans l'autre (laboratoire), elle est quasi inexistante: le locuteur ne dispose que de son système phonétique. Par ailleurs, si les systèmes étudiés présentent tous deux des états de dégradation plus avancée en parole spontanée, il convient cependant de remarquer que les phênomènes observés semblent de nature différente dans les deux langues. En particulier, la tendance à la centralisation des timbres n'est présente qu'en espagnol, sans doute du fait de la plus grande simplicité du système espagnol, qui peut mieux tolérer un phénomène d'ensemble de ce type. En français, cependant les mouvements des agrégats vocaliques semblent autrement plus complexes. En tout cas, la centralisation -qui est le phénomène le plus souvent évoqué dans la littératureapparaît n'être qu'une modalité facultative des phénomènes entropiques soulignés; en effet, d'une part, elle peut être absente de systèmes pourtant dégradés (cas du français), et d'autre part, son ampleur ne semble pas en relation directe avec la probabilité de confusion dans le chef des voyelles considérées (cas de l'espagnol). 